\title{
Power minimization of WBSN using adaptive routing protocol
}

\author{
Ammar Sabeeh Hmoud Altamimi ${ }^{1}$, Omar Raad K. Al-Dulaimi ${ }^{2}$, Amar A. Mahawish ${ }^{3}$, M. M. Hashim ${ }^{4}$ \\ Mustafa Sabah Taha ${ }^{5}$ \\ ${ }^{1}$ University of Baghdad College of Engineering, Iraq \\ ${ }^{2} \mathrm{Al}$-Iraqia University/College of engineering/Network Department, Iraq \\ ${ }^{3}$ Al-Iraqia University/College of Engineering/Computer Department, Iraq \\ ${ }^{4}$ Faculty of Engineering, Uruk University, Iraq \\ ${ }^{5}$ Basrah Oil Training Institute, Ministry of Oil, Iraq
}

\begin{tabular}{l} 
Article Info \\
\hline Article history: \\
Received Dec 5, 2019 \\
Revised Feb 2, 2020 \\
Accepted Feb 16, 2020 \\
\hline
\end{tabular}

\section{Keywords:}

Actuators

Data packet

Intelligent WBSN

Nodes

Sensors

\begin{abstract}
The present work was an attempt to address a challenge of energy utilization or consumption in WBSN, it used a proposed routing algorithm based on ant optimization technique to distribute energy utilization efficiently on nodes. Thus reducing consumed energy and extending the life cycle of nodes, as well as avoiding damages might be occurred in tissues of patient's body. At the beginning the proposed protocol was compared with conventional routing protocol LEACH to prove it's efficiency in the extending of the life cycle of node, then it was used with experimental network was employed to examine energy utilizations. The obtained results were compared with others attained by conventional and developed routing protocols, there was considerable minimizing in the energy consumption that proved efficiency of proposed algorithm.
\end{abstract}

Copyright $(2) 2020$ Institute of Advanced Engineering and Science. All rights reserved.

Corresponding Author:

Mustafa Sabah Taha,

Basrah Oil Training Institute, Ministry of Oil, Iraq.

Email: timimymustafa@gmail.com

\section{INTRODUCTION}

A contribution of telecommunication has raised tremendously in developing of diverse cutting edges applications such as intelligent cities, satellite and electronic commerce. Latest paradigms of telecommunication like Internet of things (IoT) and machine-to-machine (M2M) have played a vital role in minimizing required time and efforts to transfer data worldwide with high quality. The Internet of things (IoT) can be described as the expansion of internet connectivity into apparatuses and objects which have been used by human. The accomplishment of the Internet connection has been performed by different hardware parts such as routers, hubs and sensors, these apparatuses are responsible for communicating and interacting with others on the Internet, and they provide remote monitoring and control. The quality of (IoT) has been enhanced extremely because of rapid development fields such real embedded systems, time analytics and wireless sensor network [1-2].

Wireless sensor network (WSN) as shown in Figure 1 submits an assembly of distributed and contributed sensors in order to monitor and trace the physical circumstances of the particular environment and systematizing the accumulated data (information) involved this environment. WSNs compute diverse circumstances for instance natural phenomena such as temperature, sound or physical activities such as walk and run. At the moment WSN are utilized in various applications, for instance industrial process monitoring and control, remote medical monitoring, and others [3-4].

Globally, numerous number of people face death because of cancer, cardiovascular disease, and many dangerous infections yearly. The frequent trouble with all killer diseases is that patients suffer from some known symptoms however diagnose of disease is delayed largely. Medical researches has confirmed that the majority of illnesses can be avoided if they are diagnosed early. Consequently, future medical care 
systems must offer more efficient organization system and focus on premature diagnose in order to struggle diseases extremely. One of essential factors to obtain high quality and imperative medical care systems can be achieved by monitoring health status of patient remotely to conduct early diagnose and avoid more bad situation. Thus, dealing with human`s body as environment witnesses different conditions and changes every day is so important assumption and because there is no patient wants to stay at a hospital for ever, wireless body sensor or( area) network (WBSN) or (WBAN) is the perfect framework to face this challenge $[5,6]$.

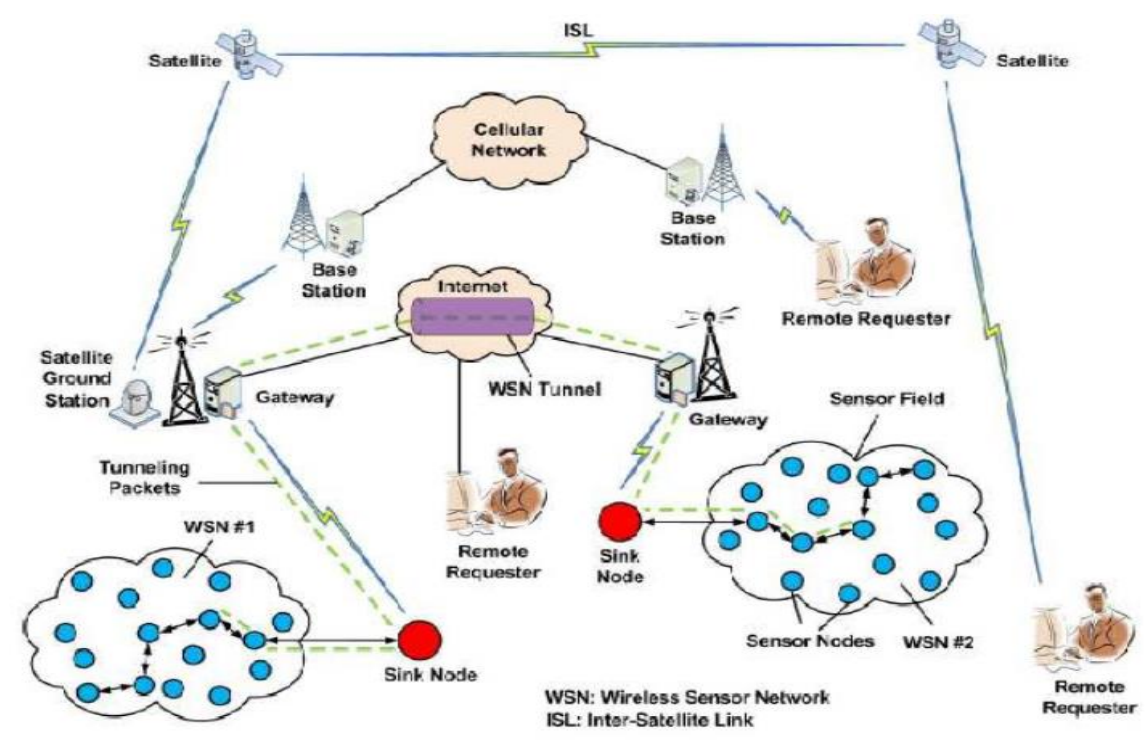

Figure 1. Comprehensive architecture of WSN [7]

Energy consumption is considered one of prior challenges involve WBSN for two reasons, first one practically it is so difficult to replace battery within components of WBSN when nodes are inserted inside patient's body. Second reason is energy consumption over entire WBSN normally leads to increase the temperature of patient's body so this might destroys tissues of body. WBSN has four distinguishable features. First one is numerous number of nodes; the second is not easy to recharge node energy and network topology is effortless to vary; as well as lack of firm control center in the network.

Some researches have focused on particular communication approaches such as cognitive cooperative in order to decrease power losses between nodes [8] whereas other works have utilized network coding to reduce power consumption [9]. [10] Proposed link forcasting technique between nodes to choose best possible path with minimum power, in spite of optimal energy routing draw extreme attention in WBSN because of short life of battery [11], some scholars have confirmed that refining the battery cells is not sufficient tool in order to handle the energy consumption in WBSN [12]. The proposed work suggests arouting algorithm (protocol) for WBSN which is based on developed Ant colony optimization. It can provide a required balance of the energy utilization of the sensor nodes and extend the lifecycle of the entire network.

\section{WIRELESS BODY SENSER NETWORK}

\subsection{Classification of WBSN}

WBSN can be defined as a wireless network of wearable computing devices. These devices may be inserted inside the body or may be external-mounted on the body in a permanent position. Such wearable devices which are monitoring health of patient can bear in private things for instance clothes, pockets or baggage. WBSNs are categorized into three types as below:

a) Managed WBSN: the work procedure of such networks can be summarized in data accumulated from one or more than one sensor nodes have been transferred and subjected to a decision of third party such as doctor, care taker or health centre. According to these data doctor for example will make a appropriate decision involves patient's health. Managed WBSN takes advantages of advanced networks such as WIFI or GSM to obtain high level of medical service reliability [13]. 
b) Autonomous WBSN: The basic function of this type is same as Managed WBSNs however the work procedure is different. In such networks actuators have been added to the sensor nodes. Thus there is no need to third party in order to help the patient, because the actuators are responsible for immediate treatment based on accumulated data during least time. This technology is considered outstanding solution for emergency cases, Autonomous WBSN does not require connection to WIFI or other networks hence avoiding problems involve transmissions and minimizing the required power or in other words saving power of battery [14].

c) Intelligent WBSN: This type is a permutation of the above two mentioned networks, it is considered cutting edge technology in the telemedicine field, Intelligent WBSN deals with more difficult conditions in which actuators are not enable to make a decision so the data will be sent to the specialist person. If the care taker such as doctor is late considerably the IWBSN take the responsibility and make the appropriate decision to tackle bad situation [15].

\subsection{Construction of WBSN}

A WBSN has been built by tiny devices that perform communication. Mostly they are defined as below:

\subsubsection{Sensor nodes}

The main duties have been implemented by sensor node include recognition of signal, in this phase the weak body`s signals are amplified and noise is eliminated then conversion signals from analog to digital form. The final phase is transmitting the collected data (signals) as packets wirelessly via transceiver to the internet. There are group of most common sensors are available worldwide, for instance ECG sensor which is used to diagnose heart disease and to evaluate the response of the heart to medicines. The second one is blood pressure sensor which measures systolic, as well as EEG Sensor which is responsible for monitoring electrical performance of brain and distinguishing any abnormal condition might happens. It is worthy to mentioning there are many number of sensors have been used for measuring diverse functions which involves human`s body such as oxygen level in blood during respiration and Glucose level in blood. Figure 2 shows basic architecture of WBSN including diverse sensors [16-17].

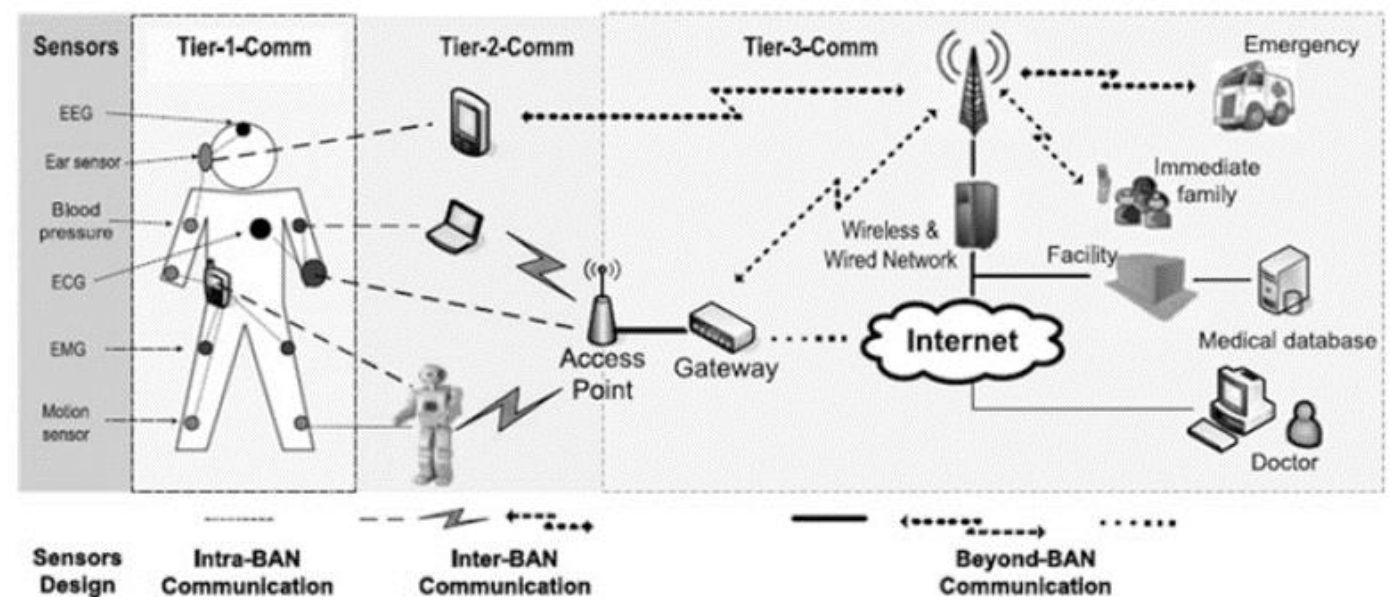

Figure 2. Basic architecture of WBSN [18]

\subsubsection{Actuator nodes}

Actuators acquire distinct quality due to their ability to help patients without waiting medical staff, under emergency conditions few seconds might be adequate to terminate patient's life so the required treatment should be offered rapidly. As mentioned previously, actuators support sensors to perform integrated medical task successfully. In addition to sensors and actuators, there are private gadgets have assemble data which have been assembled by nodes, then they transfer data throughout gateways or to the actuators on the body to carry out necessary help. Furthermore, high performance of WBSN needs to efficient software environment to accomplish its tasks sufficiently. Operating Systems represents very vital element of WBSN software and executes different jobs like duty arrangement, memory and power administrations. Many well-know operating systems used in WBSN applications such as RIOT, Lite OS, ERIKA Enterprise and others [19-21]. 


\subsubsection{Challenges of WBSN}

Different challenges have faced success of WBSN, first of all them is Quality of Service (QoS) and dependability. WBSN should be capable to transmit tremendous amount of data within short time so as to avoid delay which might cause dangerous conditions or death of patient, thus high level of quality and reliability should be available. Second one is the security and privacy of patient, authenticity of data should be preserved so as to ensure private data have not been altered at receiver party, as well as ensuring that data have been arrived securely to the authorized party [22-23].

There are other challenges involve medium access control layer (MAC) which is responsible for controlling and multiplexing data throughout the transmission medium. In addition to validating data veracity by confirming the sender's packets sequences, there is a challenge involves possibility of packets loss which is influenced by the transmission delay and the bit error rate of channel. Furthermore, packets of data are diverse; some of them are necessitated to be conveyed immediately whereas the others should be delivered dependably thus WBSN needs to efficient organization to systematize the traffic of packets [24]. The mobility of the sensor nodes might differ according to nature of network, Remote health of patients' conditions in hospitals, houses and other places have been implemented through applications have diverse network configuration depending on number of users (patients). Consequently, mobility is one of crucial issues that can influence the quality of WBSN [25].

\subsubsection{Routing protocols}

Routing protocols identify how routers communicate with each other, allocating data that enable them to pick appropriate route between two nodes on a WBSN. Routers achieve the "traffic directing" tasks on the WBSN and other networks integrate with Internet; data packets are advanced throughout the networks from router to another one until they arrive at their authorized receiver such as computer or smart phone. Routing protocols have been working to handle complicated problems; hence they are classified comprehensively into; Cross-Layered Routing Protocols, Cluster Based Routing Protocols, Postural-MovementBased Routing Protocols, Qos-Aware Routing Protocols according to the nature of problem [26-29].

\section{PROPOSED METHOD}

\subsection{Energy utilization modeling}

The energy utilization or consumption modeling might influence the benefits and performance of the protocol, the proposed research utilized energy utilization model which is described in the LEACH routing protocol so as to conduct comparison [30]. The energy utilization of broadcasting and acceptance $\mathrm{E}_{\text {elec }}=50 \mathrm{~nJ} /$ bit Unit energy utilization of broadcasting amplification $\mathrm{E}_{\mathrm{amp}}=100 \mathrm{pJ}\left(\right.$ bit $\left.\mathrm{x} \mathrm{m}^{2}\right)$ Unit energy utilization of data fusion $\mathrm{E}_{\mathrm{fuse}}=5 \mathrm{~nJ} /$ bit The energy utilization of transmitting data relies on the data size and the broadcasting distance. Whereas the energy utilization of receiving data only relies on the size of the data. Consequently, if a node send $\mathrm{k}$ bit data to another node via distance between them is $\mathrm{d}$, the energy utilization is :

$$
\mathrm{E}_{\mathrm{Tx}}(\mathrm{k}, \mathrm{d})=\mathrm{E}_{\mathrm{elec}} \times \mathrm{k}+\mathrm{E}_{\mathrm{amp}} \times \mathrm{k} \times \mathrm{d}^{2}
$$

The energy utilization of receiving is

$$
\mathrm{E}_{\mathrm{Rx}}(\mathrm{k})=\mathrm{E}_{\text {elec }} \times \mathrm{k}
$$

The energy utilization of data fusion is

$$
\mathrm{E}_{\text {fuse }}(\mathrm{k})=\mathrm{E}_{\text {fuse }} \times \mathrm{k}
$$

There are three essential assumptions have been conducted to make proposed routing protocol more comprehensive, first one the primary energy of all nodes is equal and every node can share data with its neighbor nodes finally the assumed network is very large scale application that makes it more close to practical situation as it is happened in case of large number of patients monitored by one hospital.

\subsection{The mathematical formulation of ant colony algorithm [31-32]}

The proposed reaserch utilized Ant colony algorithm which is one of meta heuristic optimization algorithms to accomplish more compatible energy distribution among nodes in WBSN. In this work, $\mathrm{n}$ city, $\mathrm{m}$ represents number of ants in the ant colony is a basic assumption as well as some definitions should be submitted to simulate the performance of real ants.

Firstly, essential notations should be defined as below: 
$\mathrm{m}$ : the number of ant in the ant colony.

$b_{i}(t)$ : number of ant of city $i$ at an instant of $t$.

$\mathrm{d}_{\mathrm{ij}}$ : distance between city $\mathrm{i}$ and $\mathrm{j}$.

$\mathrm{N}_{\mathrm{ij}}$ : visibility of edge $(\mathrm{i}, \mathrm{j})$, which explains the degree of motivation that a node moved from city $\mathrm{i}$ to $\mathrm{j}$. it is calculated by $N_{i j}=\frac{1}{d_{i j}}$

$\tau_{i j}:$ pheromone concentration of edge $(\mathrm{i}, \mathrm{j})$.

$\alpha$ : comparative significance degree of pheromone.

$\beta$ : relative importance degree of visibility.

$p_{i j}^{Z}$ : relocating probability of ant $\mathrm{z}$, where $\mathrm{j}$ is the city not yet visited.

The performance of every ant ought to be restricted by the subsequent regulations:

(1) Ant chooses following path in accordance with the pheromone amount on the path.

(2) The used path previously should not be activated latterly. It is managed by a data table record.

(3) When a cycle has been done, pheromone amount of the entire path is discharged, and pheromone on the path is updated.

Initially, all edges are granted a pheromone. Ant $\mathrm{z}(\mathrm{z}=1,2, \ldots \mathrm{m})$ decides direction in accordance with the quantity of pheromone on every path. Tabu $\operatorname{record}(\mathrm{z}=1,2, \ldots \mathrm{m})$ is employed to trace nodes which ant $\mathrm{z}$ has been moved. In the investigation procedure, condition transition probability is estimated consistent with the pheromone of each path. At the instant $\mathrm{t}$, transfer probability $p_{i j}^{z}$ is estimated by the subsequent (4).

$$
p_{i j}^{Z}=\left\{\begin{array}{cc}
\frac{\tau_{i j}^{\alpha}(t) N_{i j}^{\beta}(t)}{\sum_{s \in \text { allowed }_{z} \tau_{i s}^{\alpha}(t) N_{i s}^{\beta}(t)}} \quad, j \in \text { allowed }_{z} \\
0 & \text { otherwise }
\end{array}\right.
$$

Where :

$\tau_{i j}(t):$ the amount of pheromone.

allowed $_{z}=\left\{C-\right.$ tabu $\left._{z}\right\}$ : node that can be selected in the next.

For keeping away from the surplus remaining pheromone which flood instigated information, remaining pheromone will be computed after implementation the traversal of the entire nodes. The new amount of pheromone is computed using below equation.

$$
\tau_{i j}(t+n)=(1-\rho) \tau_{i j}(t)+\Delta \tau_{i j}(t)
$$

Where

$\square \square$ : evaporation coefficient of pheromone,

1- $\square \square$ : remaining coefficient of pheromone,

$\Delta \tau_{i j}(t)$ : expanded pheromone of edge $(\mathrm{i}, \mathrm{j})$

$$
\begin{aligned}
& \Delta \tau_{i j}(t)=\sum_{z=1}^{m} \Delta \tau_{i j}^{Z} \\
& V T_{i j}^{Z}=\left\{\begin{array}{rr}
\frac{W}{L_{z}} & \text { if ant z picks edge }(\mathrm{i}, \mathrm{j}) \text { in z cycle } \\
0 & \text {, else }
\end{array}\right.
\end{aligned}
$$

Where

$\mathrm{W}$ : represents the whole quantity of pheromone,

$\mathrm{L}_{\mathrm{z}}$ : represents span of path of ant $\mathrm{z}$ in this cycle.

\subsection{Routing algorithm derived from improved ant colony system \\ 3.3.1. Development of ant colony optimization}

Based on mentioned procedure, ant arrives at the destination via the majority of pheromone path. If a current path represents the best path to the objective, the quantity of ants in the path will be enormous. That means the node energy of the network on the path diminishes extremely and the life cycle of the entire network will be limited. Hence, ant colony algorithm should be developed to distribute the energy utilization of the nodes efficiently or in other words obtaining most possible balanced utilization. The proposed work has conducted the required above goal based on the following features which described as : 
a) Modifying Equation of State Transition Probability

$\mathrm{p}_{\mathrm{ij}}^{\mathrm{z}}$ is determined by the following formula

$$
p_{i j}^{Z}= \begin{cases}\frac{\tau_{i j}^{\alpha}(t) N_{i j}^{\beta}(t) E_{j}}{\sum_{s \in \text { allowed }_{z} \tau_{i s}^{\alpha}(t) N_{i s}^{\beta}(t) E_{S}}}, j \in \text { allowed }_{z} \\ 0 & \text { otherwise }\end{cases}
$$

Where

$\tau_{\mathrm{ij}}^{\alpha}$ : pheromone intensity

$\mathrm{N}_{\mathrm{ij}}^{\beta}$ : the converse of the space from the node $\mathrm{i}$ to node $\mathrm{j}$,

$E_{j}$ : the remaining energy of node that ant $z$ will visit,

allowed $\mathrm{z}$ represents set that ant $\mathrm{z}$ has not gone.

The above equation has taken into consideration of residual energy of node so energy organization factor is added in the (8). The energy utilization of nodes is distributed appropriately and the lifecycle of the entire network is extended. As well as the postponement factor is considered in the (8).

b) Flexibility of network configuration

The changeable network structure is intending, so ant $\mathrm{z}$ which situates in the node $\mathrm{i}$ chooses the next node in accordance:

$$
\mathrm{p}_{\mathrm{ij}}^{\mathrm{z}}= \begin{cases}\frac{1}{\mathrm{~L}_{\mathrm{n}}} & \text { if } \mathrm{q} \leq \mathrm{q}_{0} \\ \text { calculate } \mathrm{p}_{\mathrm{ij}}^{\mathrm{z}} \text { by equation(8), else }\end{cases}
$$

Where :

$\mathrm{q}:$ a arbitrary number and,

$\mathrm{L}_{\mathrm{n}}$ : the number of neighbors of node $\mathrm{n}$.

$\mathrm{q}_{0}$ : this value will determine the possibility of looking for the new path along with the pheromone or discover the new path.

Based on above equation, in case of the high value of $q_{0}$, ants simply pick edges which pheromone is large. Therefore; the suggested algorithm is effortless to find local best solution. On the other hand, the low value of $q_{0}$ makes the investigation space enlarged. However, the investigation has a sightlessness and the algorithm will not be able to converge efficiently to the best solution. The two characteristics are considered, thus the value of $q_{0}$ is vibrant modification in the iterative procedure. $q_{0}$ is decided by the below :

$$
q_{0}=\left\{\begin{array}{cc}
c & 0<\text { cycle } \leq n_{0} \\
c_{0} & n_{0}<\text { cycle } \leq n_{1} \\
c_{1} & n_{1}<\text { cycle } \leq n_{\max }
\end{array}\right.
$$

Where

$n_{0,} n_{1}$ : the number of iterative,

$n_{\text {max }}$ : the greatest number of iterative

$0.7<c<0.9$ and $0<c_{0}<0.3$

When the algorithm begins execution, edges have large pheromone are chosen simply. Subsequently iterative $n_{0}$, to facilitate keeping away from local optimal solution, $q_{0}$ is varied to increase search space. After iterative $n_{1}$, for helping the algorithm to meet the global best solution, $q_{0}$ is modified once more.

\section{c) Solving loop problem}

Like others optimization algorithms, ant colony has experienced loop problem. In order to avoid this issue, hop adding data is traced at the expense of a extremely tiny space. Under the route grounding phase, nodes which surround the sink node (assume $\mathrm{z}$ ) recognize that sink node in their neighborhood node table. The number count of these nodes will be 1 . Simultaneously, neighbors will launch their jump number data to their neighbor nodes. At what time neighbor nodes $\mathrm{z}+1$ obtain data, they will set their jump number and 
launch their jump number data to neighbor nodes. Finally, a node picks following node, the number of jump number have to be higher than itself. Thus the proposed algorithm acquired speed of convergence and stayed away from loop.

\subsubsection{The proposed routing algorithm}

Phase 1. Route Grounding.

Sink node transmits preliminary signal; every node will obtain its neighbor node also insert it to its route table. The pheromone of neighbor node link will be 1 . Additionally, every node produces recent node's ant pack which contains number of node and route table. The maximum number of iterations is set to $R_{\max }$ and the primary iteration number is set to 1 .

Phase 2. Route Organization.

Under this phase, the ant which situates in node i will pick the following node in accordance with (8), (9) and (10). At what time a node obtain the ant package, it will advance ant package based on the probability $p_{i j}^{Z}$ Phase 3. Route Optimization.

Instantaneously, sink node receives ant package, it will count number of ants pack which launched by every node. It is assumed a entire number of node is $\mathrm{m}$ and the number of ants package launched by node $\mathrm{i}$ is $\mathrm{X}_{\mathrm{i}}$ $(\mathrm{i}=1, \ldots \mathrm{m})$

The entire quantity of ant package of network calculated by:

$$
T=\sum_{i=1}^{m} x_{i}
$$

The quantity of ant packet transmission is,

$$
E(x)=\sum_{i=1}^{m} \frac{x_{i}}{T} x_{i}
$$

The variance of the amount of the ant packet is,

$$
E(x)=\sum_{i=1}^{m}\left(x_{i}-E X\right)^{2} \frac{x_{i}}{T}
$$

The assessment function of the amount of the ant packet is described by,

$$
\mathrm{F}(\mathrm{x})=\mathrm{E}(\mathrm{x}) \mathrm{D}(\mathrm{x})^{\tau}
$$

$\tau$ represents a modifiable factors.

An amendment of weight of $\mathrm{E}(\mathrm{x})$ and $\mathrm{D}(\mathrm{x})$ has been done by $\tau$, every node produces back ant package, at what time nodes receive back ant package, neighborhood link data of back ant package will be renewed using equation 15

$$
\begin{aligned}
& \tau_{i j}(t+n)=(1-\rho) \tau_{i j}(t)+\Delta \tau_{i j}(t) \\
& \Delta \tau_{i j}(t)=\frac{F(x)-F_{\min }(x)}{F(x)+F_{\min }(x)}
\end{aligned}
$$

The node which situates in the back ant package is picked as the following hop. Simultaneously, decision will be made to launch a new packet of ants in accordance with the information in the back ant packets. Route is founded in case of new ant package does not necessitate launching.

\section{RESULTS AND DISCUSSIONS}

With the purpose of evaluatation of the quality performance of the suggested routing algorithm, it was compared with LEACH algorithm. The obtained results have been computed on an Intel Core i3 2.6 GHz with 4 G RAM under a Microsoft Windows 7 and MATLAB R2013a used to conduct the required tests. At the beginning 40 nodes arbitrarily allocated in the range of $150 \times 150$. For study purpose, the estimated data in transmitting and receiving modes is 3000 bit for each node. Additionally, the base is situated in (75, 75). The primary assumed energy of every node is $0.6 \mathrm{~mJ}$.

It is essentially to carry out the high performance of the suggested routing algorithm, the values of $\alpha, \beta, \rho$ should be examined. Wide range of values have been conducted, finally the best values are $\alpha=4, \beta=$ $5, \rho=0.8$. It is worthy to mentioning these values computed via frequent trails. After this phase, the life cycle of nodes based on proposed and LEACH algorithms were compared. The value of Q is 0.6. The 
life cycle of the 1th, 3th, 5th, 8th, 12th, 15th, 18th, 23th, 27th, 31th, 35th and 40th node is examined as illustrated in Figure 3.

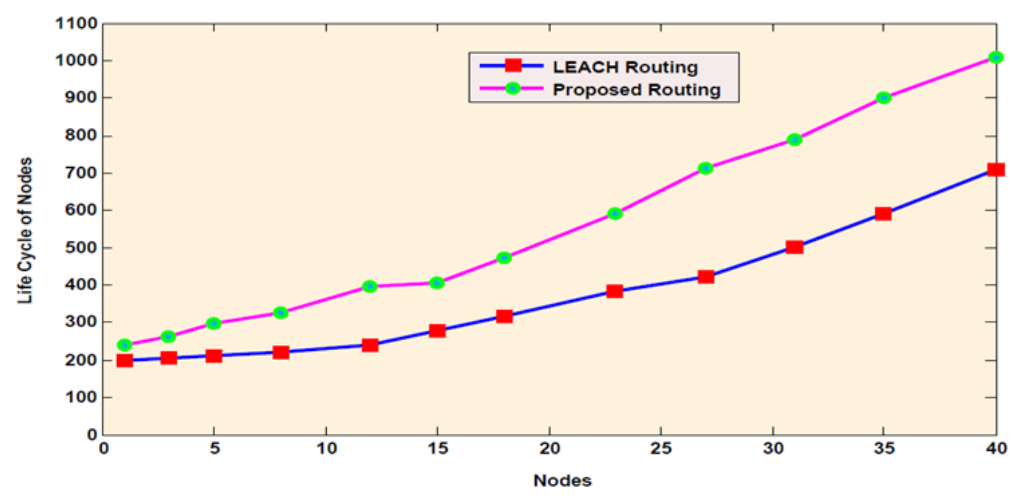

Figure 3. Life cycle of nodes

The above test has been conducted frequently to confirm the obtained results, obviously the proposed routing algorithm contributed effectively in extending the life cycle of the suggested network. Consequently, energy consumption, reliability and quality of remote health monitoring have been developed considerably with the proposed routing algorithm which was adopted from the ants behavior to optimize the best solution. After this analytical comparison the next step is conducting experimental study, Table 1 illustrates data of network was examined by two conventional and developed XL routing protocols [33].

Table 1. Data of experimental network [33]

\begin{tabular}{|c|c|c|c|c|}
\hline \multirow[t]{2}{*}{ Network parameters } & \multicolumn{2}{|c|}{ BAN-Conv protocol } & \multicolumn{2}{|l|}{ BAN-XL protocol } \\
\hline & Small network & Large network & Small network & Large network \\
\hline Number of nodes & 7 & 12 & 7 & 12 \\
\hline Number of source node & 2 & 4 & 2 & 4 \\
\hline Simulation time & \multicolumn{2}{|l|}{$5 \mathrm{~s}$} & \multicolumn{2}{|l|}{$5 \mathrm{~s}$} \\
\hline Simulation area & \multicolumn{2}{|l|}{$30 \times 30 \mathrm{~m}^{2}$} & \multicolumn{2}{|l|}{$30 \times 30 \mathrm{~m}^{2}$} \\
\hline Node's speed & \multicolumn{2}{|l|}{$0-8 \mathrm{~m} / \mathrm{s}$} & \multicolumn{2}{|l|}{$0-8 \mathrm{~m} / \mathrm{s}$} \\
\hline Data Packet size & \multicolumn{2}{|l|}{$1 \mathrm{k}$ bits } & \multicolumn{2}{|l|}{$1 \mathrm{k}$ bits } \\
\hline Traffic type & \multicolumn{2}{|l|}{ CBR } & \multicolumn{2}{|l|}{ CBR } \\
\hline Contention Window Size & \multicolumn{2}{|l|}{2} & \multicolumn{2}{|l|}{2} \\
\hline Frame Duration & \multicolumn{2}{|l|}{$15.3 \mathrm{~ms}$} & \multicolumn{2}{|l|}{$15.3 \mathrm{~ms}$} \\
\hline Control Slot duration & \multicolumn{2}{|l|}{$0.318 \mathrm{~ms}$} & \multicolumn{2}{|l|}{$0.318 \mathrm{~ms}$} \\
\hline Data rate & \multicolumn{2}{|l|}{$10-80 \mathrm{kbps}$} & \multicolumn{2}{|l|}{$10-80 \mathrm{kbps}$} \\
\hline Mobility model & \multicolumn{2}{|c|}{ Random way point (pause time $=0.1 \mathrm{~s}$ ) } & \multicolumn{2}{|c|}{ Random way point (pause time $=0.1 \mathrm{~s}$ ) } \\
\hline MAC protocol & \multicolumn{2}{|l|}{802.15 .4} & \multicolumn{2}{|c|}{802.15 .4 (improved) } \\
\hline Max back off exponential & \multicolumn{2}{|l|}{5} & \multicolumn{2}{|c|}{ 3-5 (adaptive) } \\
\hline Retrial limit & \multicolumn{2}{|l|}{4} & \multicolumn{2}{|l|}{ 3-8 (adaptive) } \\
\hline Routing protocol & \multicolumn{2}{|l|}{ AODV } & \multicolumn{2}{|c|}{ AODV (improved) } \\
\hline Control message interval & \multicolumn{2}{|l|}{$1 \mathrm{~s}$} & \multicolumn{2}{|c|}{$0.25-1$ s (adaptive) } \\
\hline
\end{tabular}

The proposed routing protocol has been employed in the above experimental network and compared with above mentioned algorithms. Sensors in the proposed network might have diverse data rate according to the patient's circumstances. The majority of serious patients require permanent monitoring of health parameters and need to sufficient reliability of transmission rates but remote monitoring of patients might be completed with less data rates. Consequently, the data rate changes within (10 -80) kbps for simulation function. In addition, the proposed protocol was examined in mobile large networks. The suggested study supposed that patients have diverse wireless sensors move freely. Results of the studied are obtained using two speeds $4 \mathrm{~m} / \mathrm{s}$ and $8 \mathrm{~m} / \mathrm{s}$.

It is noticeably from Figure 4, the proposed routing protocol achieved considerable difference in the energy consumptions of nodes WBSN. The obtained results in Table 2 confirmed exactly that the proposed 
routing protocol has positive influences on the performance of networks, in terms of extending life cycle of nodes thus increasing the network efficiency and quality of service by allocating the entire energy on nodes sufficiently. Furthermore; minimizing the consumed energy means reducing temperature that will minimize chances of damage which might be occurred in tissues of patient's body.

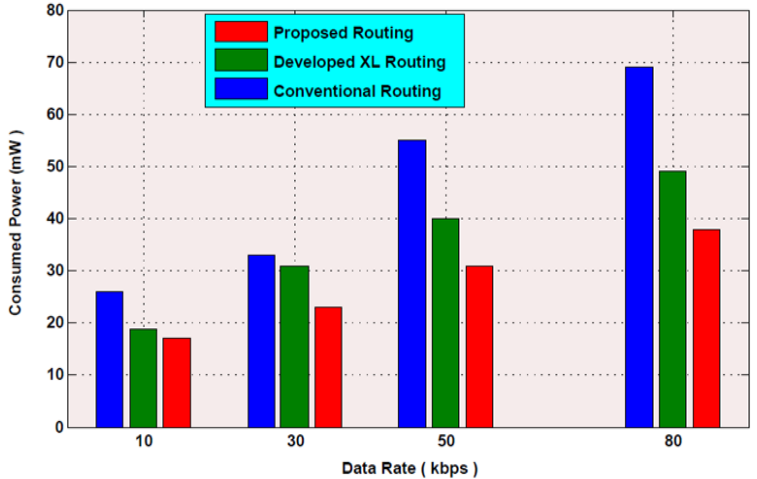

Figure 4a. Energy consumption of the experimental network with speed of $4 \mathrm{~m} / \mathrm{s}$

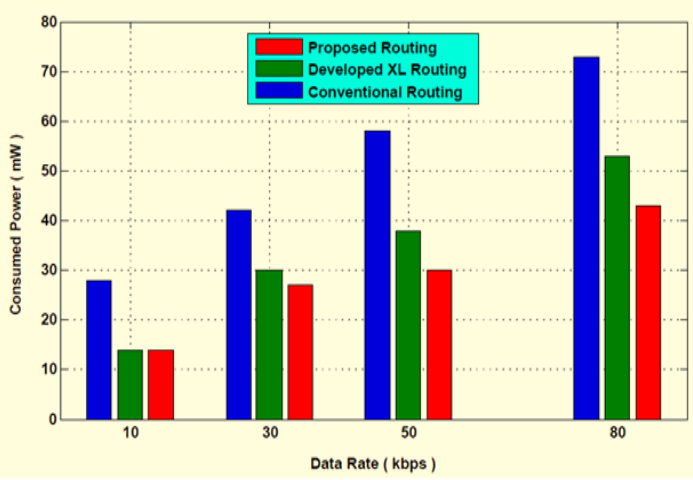

Figure 4b. Energy consumption of the experimental network with speed of $8 \mathrm{~m} / \mathrm{s}$

Table 2. Results of energy consumption of nodes

\begin{tabular}{ccccccc}
\hline $\begin{array}{c}\text { Data Rate } \\
(\mathrm{kbps})\end{array}$ & $\begin{array}{c}\text { Power Consumption }(\mathrm{mW}), \\
\text { Conventional } \\
\text { Routing }\end{array}$ & $\begin{array}{c}\text { Developed } \\
\text { XL Routing }\end{array}$ & $\begin{array}{c}\text { Proposed } \\
\text { Routing }\end{array}$ & $\begin{array}{c}\text { Power Consumption ( } \mathrm{mW}), \\
\text { Conventional } \\
\text { Routing }\end{array}$ & $\begin{array}{c}\text { Speed }(8 \mathrm{~m} / \mathrm{s}) \\
\text { XL Routing }\end{array}$ & $\begin{array}{c}\text { Proposed } \\
\text { Routing }\end{array}$ \\
\hline 10 & 26 & 19 & 17 & 28 & 14 & 14 \\
30 & 33 & 31 & 23 & 42 & 30 & 27 \\
50 & 55 & 40 & 31 & 58 & 38 & 30 \\
80 & 69 & 49 & 38 & 73 & 53 & 43 \\
\hline
\end{tabular}

\section{CONCLUSIONS}

Energy consumption challenge was addressed using proposed routing protocol based on procedure of ant colony optimization technique, the present solution tried to distribute energy consumption on nodes efficiently to extend life cycle of nodes and entire consumed energy. On the other hand, reducing the negative impact on the health of patients. Parameters of ant colony optimization algorithm affect directly on the performance of proposed algorithm that means there is a correlation between them and quality of WBSN; it can be translated mathematically for further research. Comprehensively, the performance of the suggested protocol was advanced as compared with other two.

\section{REFERENCES}

[1] Deepika N, M. Anand, F.Jerald, A. 'A novel three tier internet of things health monitoring system'. Indonesian Journal of Electrical Engineering and Computer Science, vol. 15, no. 2. pp. 631 637, 2019.

[2] Kadhim, H. A., Ali, N. S., \& Dheyaa, M. A. "Management and Achieving System for Metal Detection Robot Using Wireless-Based Technology and Online Database Registry". International Journal of Power Electronics and Drive System, vol. 10, no. 1, pp. 219-229, 2019.

[3] Devasena, G. S., \& Kanmani, S. "Robust Security for Health Information by ECC with Signature Hash Function in WBAN". IJEECS, vol. 11, no. 1, pp. 256-262, 2018

[4] Djadour, Imededine, Aidel Salih, and Hicham Medkour. "Design and implementation of low power consumption wireless sensor node." Telkomnika, vol. 17, no. 6, 2019.

[5] N. H. Ramli, Haryati Jaafar, Y. S. Lee, Hazila Othman. "Analysis of wave propagation for wireless implantable body area network application" Indonesian Journal of Electrical Engineering and Computer Science, vol. 15, no. 2. pp. 936-941, 2019

[6] S.Movassaghi, M.Abolhasan, J.Lipman, D.Smith, A. Jamalpour, "Wireless body area networks: asurvey", IEEE Commun. Surv.Tutor, vol. 16, no. 3, pp. 1658-1686, 2014, http://dx.doi. org/10.1109/SURV.2013.121313.00064.

[7] Laassiri Jalal, El Hajji Said, "Design Methodology of Energy Consumption for Wireless Sensor Networks using Renewable Energy KRIT SALAH-DDINE”, Journal Of Computing, vol. 4, no. 5, 2012,

[8] Alkhayyat, A., Thabit, A. A., Al-Mayali, F. A., \& Abbasi, Q. H. (2019). WBSN in IoT Health-Based Application: Toward Delay and Energy Consumption Minimization. Journal of Sensors, 2019. 
[9] Ali, Shahid M., et al. "Design of Compact Microstrip Patch Antenna for WBAN Applications at ISM 2.4 GHz." Indonesian Journal of Electrical Engineering and Computer Science, vol. 10, no. 1, 2018.

[10] Anwar, A., \& Duraisamy, S. "A Predictive Routing Algorithm for WBSN Based on Kalman Filter Iterations". IEEE Sensors Journal, vol. 18, no. 18, pp. 7741-7748, 2018.

[11] Mustapa, Muhammad \& Saat, Shakir \& Yusop, Yusmarnita \& Shaari, Muslimah. "Capacitive power transfer in biomedical implantable device: a review". International Journal of Power Electronics and Drive Systems (IJPEDS), vol. 10, no. 2, pp. 935-942, 2019.

[12] Sadiq, B. O., Adedokun, A. E., \& Abubakar, Z. M. "The impact of mobility model in the optimal placement of sensor nodes in wireless body sensor network". ArXiv preprint arXiv: 1801.01435, 2018.

[13] Taha, M. S., Rahim, M. S. M., Hashim, M. M., \& Johi, F. A. "Wireless body area network revisited". International Journal of Engineering \& Technology, vol. 7, no. 4, pp. 3494-3504. 2018.

[14] Sahito, A. Performance Analysis of IEEE 802.15. 6 Standard for WBSN using Castalia Simulation Tool. (2019).

[15] Pang, Z., Tian, J., \& Chen, Q. Intelligent packaging and intelligent medicine box for medication management towards the Internet-of-Things. In 16th International Conference on Advanced Communication Technology, IEEE. pp. 352-360, 2014.

[16] Sangwan, A., \& Bhattacharya, P. P. Wireless body sensor networks: a review. International Journal of Hybrid Information Technology, vol. 8, no. 9, pp. 105-120, 2015.

[17] Nur Athilah Abdul Rahman, Asral Bahari Jambek. "Biomedical health monitoring system design and analyses". Indonesian Journal of Electrical Engineering and Computer Science, vol. 13, no. 3, pp. 1056-1064. 2019.

[18] Elhayatmy, G., Dey, N., \& Ashour, A. S. "Internet of Things based wireless body area network in healthcare. In Internet of things and big data analytics toward next-generation intelligence," pp. 3-20, 2018.

[19] Alam, M. M., Arbia, D. B., \& Hamida, E. B. "Research trends in multi-standard device-to-device communication in wearable wireless networks". In International Conference on Cognitive Radio Oriented Wireless Networks (pp. 735-746). Springer, Cham, 2015.

[20] Nasri, F., \& Mtibaa, A. "Smart mobile healthcare system based on WBSN and 5G". (IJACSA) International Journal of Advanced Computer Science and Applications, vol. 8, no. 10, 2017.

[21] Tickoo, \{Vrisha, and Sapna Gambhir. "A comparison study of congestion control protocols in WBAN." Int. J. on innovations and advancements in computer science, vol. 4, no. 6, pp. 121-127, 2015.

[22] Gravina, R., Alinia, P., Ghasemzadeh, H., \& Fortino, G. "Multi-sensor fusion in body sensor networks: State-ofthe-art and research challenges". Information Fusion, vol. 35, pp. 68-80. 2017.

[23] Kim, Y. K., Wang, H., \& Mahmud, M. S. "Wearable body sensor network for health care applications. In Smart Textiles and their Applications, pp. 161-184, 2016.

[24] Shah Murtaza Rashid Al Masud, Asmidar Abu Bakar, Salman Yussof. "A modified WBANs MAC superframe using priority-criticality index table for managing pilgrims' emergency traffic in Hajj". Indonesian Journal of Electrical Engineering and Computer Science, vol. 14, no. 3, pp. 1499-1507, 2019.

[25] T.T.ThienLe, S.Moh, Interference mitigation schemes for wireless body area sensor networks: a comparative survey, Sensors, vol. 15, no. 6, 2015. 13805-13838. http://dx.doi.org/ 10.3390/s150613805

[26] Zuhra, F. T., Bakar, K. A., Ahmed, A., \& Tunio, M. A. Routing protocols in wireless body sensor networks: A comprehensive survey. Journal of Network and Computer Applications, vol. 99, pp. 73-97, 2017.

[27] Basavaraj G.N, Jaidhar C.D. "Low latency and energy efficient cluster based routing design for wireless sensor network". Indonesian Journal of Electrical Engineering and Computer Science, vol. 13, no. 2, pp. 615-625, 2019

[28] Rashid, T., Kumar, S., Verma, A., Gautam, P. R., \& Kumar, A. Pm-EEMRP: postural movement based energy efficient multi-hop routing protocol for intra wireless body sensor network (Intra- WBSN). TELKOMNIKA, vol. 16, no. 1, pp. 166-173, 2018.

[29] Abdulwahedand, Mohanad Najm, S. T. Mustafa, and Mohd Shafry Mohd Rahim. "Image Spatial Domain Steganography: A study of Performance Evaluation Parameters." 2019 IEEE 9th International Conference on System Engineering and Technology (ICSET). IEEE, 2019.

[30] S. Lindsey and C. S. Raghavendra, "PEGASIS: Power-efficient gathering in sensor information systems", The IEEE Aerosapce Conf.Montana: IEEE Aerospace and Electronic Systems Society, pp. 1125-1130, 2002.

[31] T. C. Camilo, J. S. Carreto and F. Boavida, "An energy-efficient ant-based routing algorithm for wireless sensor networks", Ant Colony Optimization and Swarm Intelligence, Proceedings, pp. 49-59, 2006.

[32] W. Hongyuan, L. Zhiyuan and B. Ying, "Ant Colony Optimization Algorithm For WSN Cross-Layer Routing Protocol”, Journal of Changzhou University, vol. 26, no. 2, pp. 32-37, 2014,

[33] Humaira Abdus Salam, Bilal Muhammad Khan., "Use of wireless system in healthcare for developing countries", Digital Communications and Networks, vol. 2, pp. 35-46, 2016. 